\title{
AN ASYMPTOTIC PROPERTY OF SOLUTIONS OF WAVE EQUATIONS
}

JEROME A. GOLDSTEIN

Let $X$ be a complex Hilbert space with inner product $\langle\cdot, \cdot\rangle$ and norm $\|\cdot\|$. Let $A$ be a selfadjoint (in general unbounded) linear operator on $X$ satisfying

$$
\langle A x, x\rangle \geqq 0 \quad \text { for all } x \in D(A),
$$

where $D(A)$ denotes the domain of $A$. We shall consider "wave" equations of the form

$$
u^{\prime \prime}(t)+A u(t)=0 \quad(t \in R)
$$

$('=d / d t)$ with initial data

$$
u(0)=f_{1} \in D(A), \quad u^{\prime}(0)=f_{2} \in D\left(A^{1 / 2}\right) .
$$

TheOREM. (i) Let $A$ be a selfadjoint operator on $X$ satisfying (1). Then for any $f_{1} \in D(A)$ and for any $f_{2} \in D\left(A^{1 / 2}\right)$, the initial value problem (2), (3) has a unique twice strongly continuously differentiable solution. Let

$$
\mathrm{II}_{f}=\left\|A^{1 / 2} f_{1}\right\|^{2}+\left\|f_{2}\right\|^{2} .
$$

Then

$$
\left\|A^{1 / 2} u(t)\right\|^{2}+\left\|u^{\prime}(t)\right\|^{2}=\Pi_{f}
$$

for all $t \in R$. Let $\left\{E_{\lambda}, \lambda \in R\right\}$ be the resolution of the identity associated with $A^{1 / 2}$. Then

$$
\lim _{|t| \rightarrow \infty}\left\|A^{1 / 2} u(t)\right\|^{2}=\lim _{|t| \rightarrow \infty}\left\|u^{\prime}(t)\right\|^{2}=\Pi_{f} / 2
$$

for all choices of initial data as in (3) if and only if

$$
\lim _{|t| \rightarrow \infty} \int_{0}^{\infty} e^{i t \lambda} d_{\lambda}\left(\left\|E_{\lambda} \cdot x\right\|^{2}\right)=0 \quad \text { for all } x \in X .
$$

(ii) If, in addition, 0 belongs to the resolvent set of $A$, let

$$
\Gamma_{f}=\left\|f_{1}\right\|^{2}+\left\|A^{-1 / 2} f_{2}\right\|^{2} .
$$

Then

$$
\|u(t)\|^{2}+\left\|A^{-1 / 2} u^{\prime}(t)\right\|^{2}=\Gamma_{f}
$$

Received by the editors February 11, 1969. 
for all $t \in R$. Moreover,

$$
\lim _{|q| \rightarrow \infty}\|u(t)\|^{2}=\lim _{|\imath| \rightarrow \infty}\left\|A^{-1 / 2} u^{\prime}(t)\right\|^{2}=\Gamma_{f} / 2
$$

if and only if (6) holds.

(iii) In order that (6) holds, it is necessary that the function $\lambda \rightarrow E_{\lambda} x$ be strongly continuous for each $x \in X$, and it is sufficient that $\lambda \rightarrow E_{\lambda} x$ be strongly absolutely continuous for each $x \in X$.

Let $\left\{F_{\lambda}, \lambda \in R\right\}$ be the resolution of the identity associated with $A$. Then $E_{\lambda}=F_{\lambda^{2}}$ for all $\lambda \in R$. Thus for $x \in X, \lambda \rightarrow F_{\lambda} x$ is strongly continuous or strongly absolutely continuous if and only if $\lambda \rightarrow E_{\lambda} x$ is.

The above theorem is a generalization of a recent result of M. Shinbrot [3]. Shinbrot proved that the strong absolute continuity of $\lambda \rightarrow F_{\lambda} x$ for each $x \in X$ was a sufficient condition for (5) and (8) to hold, and that the strong continuity of $\lambda \rightarrow F_{\lambda} x$ was a necessary condition. Condition (6) is thus the condition, weaker than strong absolute continuity of $\lambda \rightarrow F_{\lambda}$ and stronger than strong continuity of $\lambda \rightarrow F_{\lambda}$, which is both necessary and sufficient for (5) and (8) to hold.

Our proof is by semigroup methods in contrast to Shinbrot's use of spectral theoretic methods.

Proof of the theorem. Assume first that 0 is in the resolvent set of $A$. The initial value problem (2), (3) can be written as

$$
U^{\prime}(t)=M U(t), \quad U(0)=f
$$

where

$$
U(t)=\left(\begin{array}{l}
u(t) \\
u^{\prime}(t)
\end{array}\right), \quad M=\left(\begin{array}{rr}
0 & I \\
-A & 0
\end{array}\right), \quad f=\left(\begin{array}{l}
f_{1} \\
f_{2}
\end{array}\right) .
$$

The above equation will be viewed as a differential equation in the Hilbert space $Y=D\left(A^{1 / 2}\right) \times X$, equipped with the (energy) norm

$$
\left|\left(\begin{array}{l}
y_{1} \\
y_{2}
\end{array}\right)\right|=\left(\left\|A^{1 / 2} y_{1}\right\|^{2}+\left\|y_{2}\right\|^{2}\right)^{1 / 2}
$$

Then, by Lemma 3.1 of [1], $M$, with domain $D(A) \times D\left(A^{1 / 2}\right)$, is the infinitesimal generator of a strongly continuous group $\left\{T_{t}, t \in R\right\}$ of unitary operators on $Y . T_{t}$ is given by the formula

$$
T_{t}\left(\begin{array}{l}
y_{1} \\
y_{2}
\end{array}\right)=\left(\begin{array}{c}
\cosh (t B) y_{1}+\sinh (t B) B^{-1} y_{2} \\
\cosh (t B) y_{2}+\sinh (t B) B y_{1}
\end{array}\right)
$$

where $B=i A^{1 / 2}$, and $\cosh (t B), \sinh (t B)$ are defined in the obvious manner in terms of the unitary group $\{\exp (s B), s \in R\}$ generated 
by $B$. The first component of $U(t)=T_{t} f$ is thus the unique twice strongly continuously differentiable solution of (2), (3). The unitary property of $T_{t}$ proves (4), and (7) follows from

$$
\begin{aligned}
\|u(t)\|^{2}+\left\|B^{-1} u^{\prime}(t)\right\|^{2} & =\left|\left(\begin{array}{c}
B^{-1} u(t) \\
B^{-1} u^{\prime}(t)
\end{array}\right)\right|^{2}=\left|\left(\begin{array}{cc}
B^{-1} & 0 \\
0 & B^{-1}
\end{array}\right) T_{t} f\right|^{2} \\
& =\left|T_{t}\left(\begin{array}{cc}
B^{-1} & 0 \\
0 & B^{-1}
\end{array}\right) f\right|^{2}=\left|\left(\begin{array}{cc}
B^{-1} & 0 \\
0 & B^{-1}
\end{array}\right) f\right|^{2}=\Gamma_{f}
\end{aligned}
$$

for all $t \in \boldsymbol{R}$.

Note that if $V$ is any unitary operator on $X$ and if $x, y \in X$, then

$$
\|x+V y\|^{2}=\|x\|^{2}+\|y\|^{2}+2 \operatorname{Re}\langle x, V y\rangle .
$$

Now let $V=\exp (-2 t B)$. Then by (9), the definition of the norm in $Y$, and the fact that $\exp (t B)$ is unitary,

$$
\begin{aligned}
4\left\|u^{\prime}(t)\right\|^{2} & =\left\|\exp (t B)\left\{(I+V) f_{2}+(I-V) B f_{1}\right\}\right\|^{2} \\
& =\left\|f_{2}+B f_{1}+V\left(f_{2}-B f_{1}\right)\right\|^{2} \\
& =2\left(\left\|f_{2}\right\|^{2}+\left\|B f_{1}\right\|^{2}\right)+2 \operatorname{Re}\left\langle f_{2}+B f_{1}, V\left(f_{2}-B f_{1}\right)\right\rangle
\end{aligned}
$$

by (10) and the parallelogram law. Thus

$$
2\left\|u^{\prime}(t)\right\|^{2}=\Pi_{f}+\operatorname{Re}\left\langle f_{2}+B f_{1}, V\left(f_{2}-B f_{1}\right)\right\rangle .
$$

A similar calculation yields

$$
2\|u(t)\|^{2}=\Gamma_{f}+\operatorname{Re}\left\langle f_{1}+B^{-1} f_{2}, V\left(f_{1}-B^{-1} f_{2}\right)\right\rangle .
$$

Hence by (11) and (4), (5) holds if and only if

$$
\lim _{|t| \rightarrow \infty} \operatorname{Re}\left\langle f_{2}+B f_{1}, V\left(f_{2}-B f_{1}\right)\right\rangle=0
$$

for all $f_{1} \in D(A)$ (i.e. for all $B f_{1} \in D(B)$ ) and for all $f_{2} \in D(B)$. Noting that $V=\exp (-2 t B),(13)$ is thus equivalent to

$$
\lim _{|t| \rightarrow \infty}\langle\exp (t B) x, y\rangle=0
$$

for all $x, y \in D(B)$ and hence for all $x, y \in \mathrm{Cl}(D(B))=X$. Polarization shows that this is equivalent to

$$
\lim _{|z| \rightarrow \infty}\langle\exp (t B) x, x\rangle=0 \quad \text { for all } x \in X .
$$

Therefore (11), (12) and the above calculation show that (5), (14) and (8) are equivalent. If $\left\{E_{\lambda}, \lambda \in R\right\}$ is the resolution of the identity associated with $A^{1 / 2}$, then since $B=i A^{1 / 2}$, 


$$
\langle\exp (t B) x, x\rangle=\int_{0}^{\infty} e^{i t \lambda} d_{\lambda}\left\langle E_{\lambda} x, x\right\rangle,
$$

so that (14) is equivalent to (6). Thus (i) and (ii) are proved in the case that 0 belongs to the resolvent set of $A$.

For the general case in (i), let $A$ be a selfadjoint operator on $X$ satisfying (1). Let $A_{n}=A+2 n^{-1} A^{1 / 2}+n^{-2} I$, so that $A_{n}^{1 / 2}=A^{1 / 2}+n^{-1} I$. Let $B_{n}=i A_{n}^{1 / 2}$. Then if $u_{n}$ is the unique strong solution of

$$
u_{n}^{\prime \prime}(t)+A_{n} u_{n}(t)=0 \quad(t \in R)
$$

with initial data (3), then

$$
u_{n}^{\prime}(t)=\cosh \left(t B_{n}\right) f_{2}+\sinh \left(t B_{n}\right) B_{n} f_{1},
$$

as was shown previously. Since

$$
\exp \left(t B_{n}\right)=e^{i t / n} \exp (t B)
$$

where $B=i A^{1 / 2}$, it follows that as $n \rightarrow \infty, u_{n}^{\prime}$ converges uniformly on compact intervals to a necessarily strongly continuous function $v$ given by

$$
v(t)=\cosh (t B) f_{2}+\sinh (t B) B f_{1} .
$$

Let $u(t)=\int_{0}^{t} v(s) d s+f_{1}$. Then

$$
u_{n}(t)=\int_{0}^{t} u_{n}^{\prime}(s) d s+f_{1} \rightarrow u(t)
$$

as $n \rightarrow \infty$ uniformly on compact intervals. Hence

$$
u^{\prime \prime}(t)=r^{\prime}(t)=\sinh (t B) B f_{2}+\cosh (t B) B^{2} f_{1}
$$

since $f_{1} \in D(B), f_{2} \in D\left(B^{2}\right)$ by (3). Therefore $u$ is twice strongly continuously differentiable and

$$
\begin{aligned}
u^{\prime \prime}(t)+A u(t)= & \sinh (t B) B f_{2}+\cosh (t B) B^{2} f_{1} \\
& -B \int_{0}^{t}\left\{\cosh (t B) B f_{2}+\sinh (s B) B^{2} f_{1}\right\} d s=0
\end{aligned}
$$

since $\int_{0}^{t} \exp (s B) x d s \in D(B)$ for all $x \in X$ and $B \int_{0}^{t} \exp (s B) x d s$ $=\exp (t B) x-x$. Moreover, (4) holds. Thus $u$ is the unique solution of the initial value prcilem (2), (3), the uniqueness being a consequence of (4) and linearity.

Let $V=\exp (-2 t B)$ as before. Then from (15) it follows that (11) holds, by the same proof as before. Thus (5) holds if and only if (13) holds. Since our proof of the equivalence of (13), (14), and (6) did 
not require that $A$ be invertible, we conclude that (5) and (6) are equivalent.

We remark that it is tempting to try to deduce the general case of (i) from the special case that $A$ is invertible by applying the TrotterKato semigroup convergence theorem [4], [2], [5] to the groups

$$
T_{t}^{(n)}=\left\{\exp \left(t\left(\begin{array}{cc}
0 & I \\
-A_{n} & 0
\end{array}\right)\right), t \in R\right\},
$$

where $A_{n}^{1 / 2}=A^{1 / 2}+n^{-1} I$. However, the hypotheses of the TrotterKato theorem are not satisfied in this case since $\sup _{n, t}\left|T_{t}^{(n)}\right|=\infty$, where $|\cdot|$ is any norm which makes $Y=D\left(A^{1 / 2}\right) \times X$ into a Banach space.

It remains to prove (iii). If $\lambda \rightarrow E_{\lambda} x$ is not strongly continuous on $R$ for each $x \in X$, then $A^{1 / 2}$ has an eigenvalue $\lambda$. Let $x$ be a corresponding (nonzero) eigenvector. Then $\exp \left(i t A^{1 / 2}\right) x=e^{i t \lambda} x$, and so

$$
\left\langle\exp \left(i t A^{1 / 2}\right) x, x\right\rangle=e^{i t \lambda}\|x\|^{2},
$$

which does not converge to 0 as $|t| \rightarrow \infty$; hence (14) fails to hold and so (6) fails to hold.

Finally, let $g_{x}(\lambda)=\left\|E_{\lambda} x\right\|^{2}$. Suppose that $g_{x}$ is absolutely continuous for each $x \in X . g_{x}$ is also monotone nondecreasing and bounded. Hence its derivative, $g_{x}^{\prime}$, belongs to $L^{1}(0, \infty)$. Therefore for each $x \in X$,

$$
\int_{0}^{\infty} e^{i t \lambda} d_{\lambda}\left(\left\|E_{\lambda} x\right\|^{2}\right)=\int_{0}^{\infty} e^{i t \lambda} g_{x}^{\prime}(\lambda) d \lambda \rightarrow 0
$$

as $|t| \rightarrow \infty$ by the Riemann-Lebesgue lemma, and so (6) holds. This completes the proof.

\section{REFERENCES}

1. J. A. Goldstein, Time dependent hyperbolic equations, J. Functional Analysis 3(1969).

2. T. Kato, Remarks on pseudo-resolvents and infinitesimal generators of semigroups, Proc. Japan Acad. 35 (1959), 467-468.

3. M. Shinbrot, Asymptotic behavior of solutions of abstract wave equations, Proc. Amer. Math. Soc. 19 (1968), 1403-1406.

4. H. F. Trotter, Approximation of semi-groups of operators, Pacific J. Math. 8 (1958), 887-919.

5. K. Yosida, Functional analysis, Springer-Verlag, Berlin, 1965.

TUlane University 